\title{
COMMUNICATION
}

\section{Apport de la greffe gingivale épithélio conjonctive dans la reconstruction palpébrale}

\author{
C. Landric (Pau), C. Alande (Bouliac), M. Ndiaye (Pau)
}

La reconstruction palpébrale fonctionnelle et esthétique implique une réhabilitation de tous les plans palpébraux. Elle se base sur la distinction de deux entités fonctionnelles: la lamelle postérieure qui comporte le tarse, la conjonctive et les rétracteurs qui assurent l'abaissement de la paupière dans le regard vers le bas; la lamelle antérieure cutanéo- musculaire qui assure l'occlusion palpébrale. Toute technique doit impérativement restaurer les deux lamelles fonctionnelles. La réhabilitation du plan postérieur tarsoconjonctival peut utiliser différents greffons autologues: identiques (greffes et lambeaux tarsoconjonctival), soit analogues (greffes cartilagineuses, chondro muqueuses ou fibro muqueuses). Le recouvrement cutané dépend lui de la situation de la perte de substance et de la paupière concernée. L'utilisation de la fibromuqueuse palatine en chirurgie palpébrale fut décrite pour la première fois par Siegel en 1985. Histologiquement, le greffon palatin comporte trois couches de la superficie à la profondeur : la couche muqueuse, épithélium malpighien kératinisé, la couche glandulaire sous-muqueuse et le périoste. Cette technique à trouvée son application au centre hospitalier de Pau, dans la reconstruction de la paupière inférieure d'une patiente, après exérèse carcinologique d'un carcinome basocellulaire du bord libre. Egalement dans la reconstruction d'un plan tarso conjonctival chez un patient enophtalme pour permettre un soutien de la prothèse oculaire. Ces chirurgies sont réalisées en doubles équipes afin de mutualiser les compétences. La fibromuqueuse palatine trouve une indication de choix dans la reconstruction de tout ou partie de la lamelle postérieure de la paupière inférieure. Elle est indiquée en seconde intention pour la paupière supérieure car le taux de complications semble y être plus élevé (Leibovitch 2006). Il est nécessaire de prélever un greffon $10 \%$ plus large que la taille du défect muqueux car la greffe palatine se rétracte légèrement à moyen terme (Hatoko 1999). La fibromuqueuse palatine offre de nombreux avantages : une incurvation adéquate « moulant » le globe oculaire, une rigidité satisfaisante, une excellente survie en association à un lambeau et une morbidité acceptable au niveau du site donneur. Cohen et Shorr ont également montré que la muqueuse palatine subit une métaplasie en épithélium non kératinisé limitant ainsi à terme les risques d'irritation cornéenne et de kératite. Par opposition aux greffes tarso conjonctivales, l'intérêt majeur est la préservation de la paupière antagoniste. Les inconvénients sont surtout liés aux complications possibles: un saignement palatin souvent précoce dans les $48 \mathrm{~h}$ postopératoires (10\% des cas) (Wearne 2001); un retard de cicatrisation; une infection palpébrale à Candida; une sécrétion salivaire palpébrale due à la persistance de glandes salivaires dans le greffon et qui peut être tarie par cryothérapie. 